Contents List available at RAZI Publishing

Malaysian Journal of Geosciences

Journal Homepage: http://www.razipublishing.com/journals/malaysian-journal-of-geosciences-mig/

https://doi.org/10.26480/mjg.01.2017.32.33

\title{
Psychological Debriefing Intervention: From the Lens of Disaster Volunteers
} Siti Rozaina Kamsani,Nabisah Ibrahim,Noor Azniza Ishak

Universiti Utara Malaysia, Kedah Correspondence: rozaina@uum.edu.my This study is funded by FRGS-Flood Disaster 2015

This is an open access article distributed under the Creative Commons Attribution License, which permits unrestricted use, distribution, and reproduction in any medium, provided the original work is properly cited.

\section{ARTICLE DETAILS}

Article history:

Received 22 January 2017

Accepted 03 February 2017

Available online 05 February 2017

Keywords:

volunteers, psychological debriefing, volunteers' attributes, post disaster management

\begin{abstract}
The experience of posttraumatic stress is a sign of discomfort feeling and miserable situation especially for flood survivors. Being a first responder to the victims, the disaster volunteers are not only support providers for moral and psychological services, but also agents to reduce disaster-related-stress. Thus, the purpose of this study was to identify the key experiences of volunteers as the first responder in using psychological debriefing intervention with disaster victims. There were 20 volunteers from different agencies involved in this study. The semi-structured interview sessions were utilized for data collection. Based on the thematic analysis process, the findings indicated that the volunteer's resilience skill, emotional stability, and social altruism have been found to be the major volunteers' attributes in conducting psychological debriefing intervention. Volunteers' suggestions on implementation the psychological debriefing intervention for Malaysian context was also provided
\end{abstract}

\subsection{INTRODUCTION}

Flood disaster has emotionally impact on the mental health and stability of the primary victims. Numerous researches have stated that people who are exposed to this traumatic event mostly experience intense fear, helplessness, or horror (Talbott, 2009). Caught off guard and "numb" from the impact of a critical incident, individuals, and communities are often ill-equipped to handle the chaos of such a catastrophic situation. Providing a support and an assistance for survivors to deal with undesirable situation are significantly important among disaster volunteers as to help the survivors to continue with their lives. There are various supports that might be useful for the survivors such as psychological aspects, emotional stability, products, and monetary donations. Indeed, the psychological support has been found to be an important factor to help the survivors in overcoming and dealing with the traumatic experiences rather than other aspects.

The psychological debriefing was initially described as a critical incident stress debriefing (CISD) by Jeffery Mitchell in 1983. Bisson, Jenkins, Alexander, and Bannister (1997) defined that psychological debriefing (PD) as a set of procedures, which include counselling and information given, which aim at preventing psychological morbidity and aiding recovery after a traumatic event. The purpose of PD is to inhibit the development of post-traumatic stress disorder and other negative sequel (Cooper, 2003). Conducting the PD is not just a simple procedure as individual or group therapy, but it needs more attention and sometimes it is considered as a complex process of early intervention given by the volunteers during or after the incidents. Previous research found that the PD intervention has significantly reduced the negative effect of traumatic event (Mitchel \& Everly, 1996). However, the focus on the implementation is based on the lens' of emergency responders from North America (Dyregrov, 1997). Thus, the volunteers' responses from different cultural background are necessary and needed to be highlighted in order to identify the significant elements for conducting the PD in other cultures.

\section{Literature Review}

Individuals who are involved in the volunteer service can widely benefit not only to the community but also to the individuals who involve in it Surprisingly, little attention has been paid to the actual consequences of volunteer service for individuals' physical and/or psychological well-being (Thoits \& Hewitt, 2001). The volunteers are the first responders to deal with the survivors, they need to be aware about their physical, psychological, and emotional stability while working with survivors in any traumatic and stressful events. Sometimes the critical incidents may produce a stressful impact on the survivors, in turn, it is sufficient to overwhelm an individual's sense of control, connection, and meaning in his/her life (Pietrantoni \& Prati, 2008). In fact, each volunteer is required to be conscious on what kinds of attributes or skills that are desirable to provide in his or her service assistance. In this regard, the most important element for disaster volunteers is to prepare themselves with the basic personal preparation and readiness in dealing with the vulnerable people and situations.

The disaster volunteers are eager and excited to demonstrate their social involvements along with their physical or emotional aspects, which might be important to the volunteer work. The researchers argued that individuals' personal resources and well-being may facilitate volunteers' involvements in the volunteer work and subsequently enhance their commitments while working with any critical incidents and events. As mentioned by Allen and Rushton (1983), the volunteer's participation is highly demonstrated in individual who has a higher level of internal locus of control, self-esteem and greater emotional stability. Their findings also showed that people who are generally have greater personal coping resources (e.g., high self-esteem or an internal locus of control) and who are in better mental health might be more likely to be involved in any volunteer services. Therefore, the purpose of this paper was to identify some personal attributes and skills through the lens of Malaysian disaster volunteers

\section{Methodology}

This study employed a qualitative research design, conducted on 20 volunteers from different agencies such as counsellors, social workers, NGOs officers, and welfare officers. All of these volunteers had experienced working with Kelantan's flood survivors at least for a period of one month. Based on the Crisis Intervention Theory (Lindermann, 1944), the semistructured interview questions were designed according to volunteers' personal background, flood involvement experiences, psychological debriefing exposures, and personal reflections while working with survivors.

All volunteers were gathered in a large group and requested to respond to the informed consent as to allow the researchers to record the sessions conducted with them. Then, the volunteers were divided into two (2) groups in which each group was facilitated by one researcher. The interview session lasted for about 60 to 90 minutes. The questions were designed on six subtopics related to volunteers' flood disaster experiences while conducting a psychological debriefing intervention. Data were analyzed by using NVivo software and there were some themes that emerged from the data collected, which related to the preparation of pyschological debrieifng intervention. Findings and Discussions

Several studies found that there are some of criteria such as - values, religiosity, value of altruism, and resilience - are needed by the volunteers in providing their services to the community (Dury, De Donder, De Witte, Buffel, Jacquet, \& Verte, 2015; Pietrantoni \& Prati, 2008). In line with the previous study, the researchers have identified several themes that were arisen from the present study. Volunteers' resilience skills, emotional stability, and social altruism were emerged as some personal attributes and skills that are necessary for disaster volunteers in conducting the PD intervention among survivors.

Resilience Skills

Being the first responder for disaster survivors, the resilience skill is one of the required skills needed by the volunteers. It reflects the ability to response positively with circumstances, which in turn challenges their stabilities and functioning. The resilience can be defined as an individual's ability to cope and deal with highly disruptive situations, such as death, disaster, vulnerable, and traumatic events (Bonanno, 2004). 
). In this study, the volunteers described that they were experienced with a good level of satisfaction while conducting the PD intervention in which they were not affected by traumatic stress and experiences from the survivors. Dealing with the same experience with the other victims, it would be helpful for the volunteers to develop their resilience skills. Consequently, it helps them to feel more empathy towards the survivors. For example, Volunteer A and $\mathrm{B}$ mentioned about their experiences as flood survivors, which helped them to understand the survivors' feelings and conditions.

"being a flood survivor in 2000, and now I'm a volunteer and work with flood survivors, so it helps me to understand their feelings..." (Excerpt Volunteer A)

This is supported by the excerpt from Volunteer B, who said that:

"During that time, my family was also in the same situation, and I couldn't help

them and keep thinking of their situations...my worry was very high - I tried to

focus on the task given and get more food for the survivors..."

In fact, the resilience skill is found as an important element among the disaster volunteers due to their personal and social resources that could protect their healthy lives. Their abilities to cope with the traumatic and stressful events would be helpful for them in controlling their unstable feelings and physical conditions. Working with the disaster survivors is highly exposed to depression, it sometimes challenges the volunteers patience and burn-out. In addition, previous research found out that the volunteers' sense of belonging to the community where they live and work at is an important factor to help them to be strong in helping the disaster survivors (Pietrantoni \& Prati, 2008). It is considered as a positive adaption in facing any critical incidents, especially, a natural disaster.

\section{Emotional Stability}

Experiencing the disaster or any traumatic events will leave some emotional effects not only on the survivors, but also on the volunteers or whoever are dealing with such situations. Given a specific period of time working with the flood survivors, most of the volunteers mentioned that their emotions were stable even though it was very hard for them to express their real feelings. They were trying to control and express their frustrations, tiredness, give up, and anger, while conducting the PD intervention with the survivors. The volunteers also explained that they have tried to show their empathy instead of sympathy, in order to understand the real feeling of the survivors.

The group process was involved in PD intervention whereby the volunteers aimed at instilling the value of hope on the survivors. In this case, condemn and judgmental on survivors' situations and conditions were applicable. Besides, they were able to control themselves from being negative towards survivors' behaviors and responses, and always be patient with them during assistance, as like what the Volunteer D mentioned that:

"I was scolded and insulted by the survivors when my rescue boat had flipped over and threw out one of the kid into the flood during evacuation. At that time I just jumped into the flood and saved the kid. After that incident, I met again with that survivor and he came to me and hugged me. He said thank you for your help..."

In addition, the Volunteer $\mathrm{C}$ also explained on what he felt when he saw the water. It was a hard time for him to choose either to fulfill his duty as volunteer or go back and evacuated his family members. He described that his emotion was not stable during that time, but he was able to control his emotion and focus on his at that particular time. This scenario reflected that the emotional stability of disaster volunteer significantly influenced their services especially while working with the survivors. The stability of emotions and feelings would be helpful for disaster volunteers while working with survivors because it could reduce their pains and frustrations particularly on the incidents. Besides, the volunteers could be more patient and calmer in facing any vulnerable situations in future.

\section{Social Altruism}

Helping behavior is a value which relates to individual's personal attribute. In fact, the volunteering activity can be considered as one of the social contribution in leading to psychological well-being and it is referred to as other-oriented views of the self and significant involvement in the world (Kahana, Bhatta, Lovegreen, Kahana, \& Midlarsky, 2013). In fact, the social altruism can be defined as a concern about others' welfare, behavior, and commitments to help, often at a personal cost (Hartenian \& Lily, 2009). In this study, the researchers found that most of the volunteers were able to commit themselves in giving an aid to the survivors and they were scarified their time to stand-by whenever they were needed.

As a first responder, the attribute of social altruism might be necessary in assisting the volunteers working with the survivors. The values of social and protection were incorporating with the sense of volunteering involvement among the volunteers in this study. The result found that most of the volunteers were able to strengthen their social roles, not only as a welfare co-worker, but also as a disaster volunteers. In fact, these different roles would be helpful for them to alleviate the feeling of guilt especially about the circumstances of others. As mentioned by two volunteers:

"I tried to be patient with myself and believe if I keep thinking with what's going on, it will distract my attention on what am I doing - to help people in here..."

(Excerpt Volunteer E)

"we worked as a team and helped each other...need to find a safe location for the survivors and somehow we broke the hospital gates and few other places - work hard to provide enough food to survivors and safe place for them..."

(Excerpt Volunteer F)

Thus, having a sense of helping others, it would enhance a personal motivation in enhancing the desire to continue the volunteer participation as to provide services towards survivors. In fact, the social altruism is a vital element in promoting a desired motivation to engage in the volunteer work in community setting.

\section{Conclusion}

Conducting a psychological debriefing intervention is one of the contributions of the disaster volunteers to help the survivors. The disaster volunteers developed their resilience skills while working with the survivors. In fact, the emotional stability had made the disaster volunteers to understand the feelings of the survivors in relation to painful experiences. In addition, the social altruism is a sense of helping other, which is necessary to motivate the volunteers' participation in any volunteer activities. Therefore, with these three elements, the service of the disaster volunteers will be improved sincerely and willingly.

\section{References}

Allen, N., \& Rushton, J. P. (1983). Personality characteristics of community mental health

volunteers: a review. Journal of Voluntary Action Research, 12, 36-49

Bisson, J. I., Jenkins, P. L., Alexander, J., \& Bannister, C. (1997). Randomised controlled trial of psychological debriefing for victims of acute burn trauma. The British Journal of Psychiatry, 171(1), 78-81.

Bonanno, G. A. (2004). Loss, trauma, and human resilience: Have we underestimated the human

capacity to thrive after extremely aversive events? American Psychologist, $59,20-28$

Cooper, A. (2003). Psychological Debriefing: Theory, Practice and Evidence. Edited by

Beverley Raphael and John P. Wilson. New York, Cambridge University Press 2000, 376

pp, ISBN 0-521-64700-2. Australasian Journal of Paramedicine, 1(1). Retrieved from

http://ro.ecu.edu.au/jephc/vol1/iss1/17

Dury, S., De Donder, L., De Witte, N., Buffel, T., Jacquet, W., \& Verte, W. (2015). To volunteer

or not: The influence of individual characteristics, resources, and social factors on the

likelihood of volunteering by older adults. Nonprofit Voluntary Sector Quarterly, 44(6), 1107-1128.

Dyregrov, A. (1997). The process in Psychological Debriefings. Journal of Traumatic Stress,

$10(4), 589-685$

Hartenian, L. S., \& Lily, B. (2009). Egoism and commitment: A multidimensional approacj to

understanding sustained volunteering. Journal of Managerial Issues, 21(1), 97-118.

Kahana, E., Bhatta, T., Lovegreen, L. D., Kahana B., \& Midlarsky, E. (2013). Altruism,

helping, and volunteering: Pathways to well-being in late life, Journal Aging Health, 25(1), 159-187.

Mitchell, J. T., \& Everly, G. S. (1996). Critical incident stress debriefing. Journal of Emergency

Medical Services, 8, 36-39.

Pietrantoni, L. \& Prati, G. (2008). Resilience among first responders. African Health Sciences, 8,

514-520.

Talbott, W. R. (2009). Early Mental Health Interventions Following Disasters: What is the

Standard of Practice?. Texas Public Health Journal, 612(2), 40-41.

Thoits, P. A, \& Hewitt, L. N. (2001). Volunteer work and well-being. Journal of Health and

Social Behavior, 42(2), 115-131. 